\title{
Características acústicas de las vocales del español de Chile producidas por sujetos residentes en la ciudad de Santiago
}

\author{
Acoustic Characteristics of the Chilean Spanish \\ Vowels Produced by Subjects Resident in the City \\ of Santiago
}

Susane Díaz A.

Fonoaudióloga

Magíster en Audiología

Departamento de Salud, Universidad

de Los Lagos

\section{Pamela Cisternas L. \\ Fonoaudióloga \\ Magíster en Audiología \\ Clínica Arauco Salud}

\section{Ilse López B.}

Metodóloga

Licenciada en Estadística de Salud Facultad de Medicina, Universidad de

Chile

Contacto con el autor: Susane Díaz A. Alcalde Fuchslocher 1305, Osorno Osorno - Chile

Tel: (56 64) 2333001

Correo-e: susane.diaz@ulagos.cl

Recibido: 09/01/2015

Aceptado: 06/11/2015

\section{RESUMEN}

El presente estudio tuvo por objetivos describir las características acústicas de las vocales aisladas en jóvenes chilenos residentes en Santiago, comparar en ellos los resultados obtenidos para hombres y mujeres y también realizar una comparación con datos de hablantes en Río de la Plata (Argentina) y de Concepción (Chile). Se seleccionó una muestra de 117 estudiantes universitarios en quienes se descartó patología vocal y auditiva. Se registraron las voces de los sujetos produciendo las vocales aisladas $y$, posteriormente, se analizaron sus registros vocales con el software PRAAT. En general, en las comparaciones se advierte que en los sujetos de Santiago las mujeres presentan mayor frecuencia que los hombres en los formantes analizados (F0, F2, F3 y F4) excepto en F1. A su vez, las mujeres y hombres de Santiago evidencian una FO mayor que sus pares rioplatenses, Por último, no se advierten diferencias importantes en la comparación con hablantes de Concepción. En cada comparación, se comentan también en forma detallada los formantes de cada una de las vocales.

Palabras clave: vocales, acústica, formantes.

\begin{abstract}
The aim of this study was, firstly, to describe the acoustic characteristics of isolated vowels produced by Chilean young people from Santiago, Chile, secondly, to compare the results obtained between men and women and, finally, to compare the data with information collected from Argentinian speakers (Rio de la Plata) and Chilean speakers (Concepción). 117 university students were selected for this study. Neither vocal nor auditory pathologies were found in the participants. Vowels produced by the participants were registered in isolation and they were analyzed using the software PRAAT. In general, the comparison shows that women from Santiago produce higher frequency than men in the analyzed formants (F0, F2, F3 y F4), except in F1. It was also observed that women and men from Santiago show a higher FO formant than speakers from Rio de la Plata. Finally, no differences were found between speakers from Santiago and speakers from Concepción. In each comparison, a detailed description of the vowel formants is also presented.
\end{abstract}

Keywords: vowels, acoustic, formants. 


\section{Introducción}

En Chile se han realizado diversos estudios acústicos de las vocales entre los que se encuentra el análisis espectrográfico efectuado por León (1998), que aborda específicamente la dispersión auditiva de las vocales anteriores en la ciudad de Concepción. También se cuenta con el trabajo de Soto Barba (2007) acerca de la variación del F1 y del F2 en las vocales del español urbano y rural de la provincia de Ñuble. Por su parte, las investigaciones de Brain, Cepeda y Barrientos (1992a) aportan con un análisis sonográfico de /e/ en sílaba trabada en un estrato alto de Valdivia y con análisis espectrográfico de /a/ en el español de Valdivia (Brain, Cepeda y Barrientos, 1992b).

También en Valdivia se ha efectuado un análisis fónico del español considerando los segmentos vocálicos átonos y tónicos (Urrutia, 1976) y un análisis sonográfico de los alófonos de /e/ en sílaba abierta en el estrato alto (Cepeda, Barrientos, y Miranda, 1991).

Por otra parte, en Argentina, Aronson, Rufiner, Furmanski y Estienne (2000) han estudiado las características acústicas de las vocales del español rioplatense.

Como se puede apreciar los estudios antes expuestos se realizaron en zonas geográficas diferentes, en estratos socioculturales dispares y abordando distintos aspectos puntuales, lo que dificulta una visión global respecto de las características de las vocales. Por ello, se consideró factible aportar en la temática describiendo las características acústicas de las vocales aisladas en jóvenes chilenos, y comparar los resultados obtenidos con otros estudios como los antes mencionados. Por otra parte, resultaba de interés también aportar con valores de las características acústicas de las vocales producidas por hombres y mujeres chilenos, contribuyendo así al conocimiento acerca del tema, el que puede ser útil en los ámbitos de la fonética y de la fonoaudiología.

De acuerdo con lo anterior, en el presente trabajo se plantean los siguientes objetivos: a) determinar los valores de los cuatro formantes de las cinco vocales, emitidas en forma aislada, para hombres y mujeres en una muestra de estudiantes universitarios chilenos; b) determinar diferencias porcentuales entre los resultados obtenidos en los universitarios chilenos, y los obtenidos en hablantes de Argentina; y c) comparar los resultados de este estudio con los descritos en Concepción

\section{Método}

Se utilizó un diseño descriptivo, con enfoque cuantitativo y diseño cuasi experimental.

Como variables se consideraron los valores de los formantes (F0, F1, F2, F3 y F4) de las cinco vocales en forma aislada y el género masculino y femenino.

\section{Participantes}

Para la elección de las unidades de estudio, se analizaron 117 grabaciones de audio digital de 117 estudiantes (39 hombres y 78 mujeres) de diversas carreras de la Universidad Mayor, con edades comprendidas entre los 17 y los 26 años de edad, que no presentaban patología vocal ni déficit auditivo. Para descartar alguna patología vocal, se realizó un análisis clínico perceptual y un análisis 
fonético acústico con el programa PRAAT; mediante audiometría tonal se eliminó a los alumnos que presentaban alguna patología auditiva.

\section{Procedimientos}

Los instrumentos para la recolección de datos fueron un informe de evaluación clínica vocal y audiometría de los sujetos, además del análisis de los resultados obtenidos con el software PRAAT y archivos de audio digital de cada sujeto.

Se estudiaron las vocales de forma aislada, con el fin de obtener las características acústicas de ellas en cada sujeto. La duración de la emisión de las vocales varió entre 1 y 2 segundos. El análisis individual de cada vocal se realizó reduciendo al máximo el fenómeno de coarticulación y analizando el segmento estable de la fonación.

El registro de las voces se efectuó utilizando los micrófonos Shure SM 48 Dynamic Low-Z Cardioide unidireccional y Shure RS 25 unidireccional, los cuales se ubicaron a una distancia de $10 \mathrm{~cm}$. Además, se usó una tarjeta de sonido Realtek HD audio output. Se les solicitó a los sujetos inspirar previamente a la emisión de los fonemas y siempre que lo necesitaran; luego se analizaron los registros vocálicos extrayendo los valores de F0, F1, F2, F3, F4 en los participantes de la muestra. Se buscó el segmento vocálico más estable y con mayor dispersión aplicando zoom hasta que se visualizaban 10 ciclos de fonación; al llegar a este momento se seleccionaba un punto y se extraía el valor del formante a estudiar.

\section{Resultados}

Los valores de los cuatro formantes de las cinco vocales en forma aislada, para hombres y para mujeres de Santiago de Chile se presentan en las Tablas $1,2,3,4$ y 5 .

Tabla 1

Promedios y desviaciones estándar (DE) de FO, F1, F2, F3 y F4 en la vocal /a/, en hombres y mujeres de Santiago de Chile

\begin{tabular}{ccccc}
\hline & & \multicolumn{3}{c}{ Vocal /a/ } \\
\hline $\begin{array}{c}\text { Característica acústica } \\
\text { de la voz }\end{array}$ & Promedio & $D E$ & Masculino \\
\cline { 2 - 5 } & 217 & $\pm 23,56$ & 140 & $D E$ \\
\hline F0 & 697 & $\pm 270,9$ & 812 & $\pm 28,07$ \\
F1 & 1597 & $\pm 220,5$ & 1332 & $\pm 94,1$ \\
F2 & 2994 & $\pm 247,7$ & 2774 & $\pm 176,6$ \\
F3 & 4301 & $\pm 251,2$ & 3986 & $\pm 253,3$ \\
F3 & & & & $\pm 283,9$ \\
\hline
\end{tabular}

Tabla 2

Promedios y desviaciones estándar (DE) de FO, F1, F2, F3 y F4, para la vocal /e/, en hombres y mujeres de Santiago de Chile

\begin{tabular}{ccccc}
\hline & \multicolumn{3}{c}{ Vocal /e/ } & \multicolumn{2}{c}{ Masculino } \\
\hline $\begin{array}{c}\text { Característica acústica } \\
\text { de la voz }\end{array}$ & Promedio & Demenino & Promedio & $D E$ \\
\cline { 2 - 5 } F0 & 216 & \pm 17.84 & 164 & \pm 48.49 \\
F1 & 486 & \pm 130.6 & 474 & \pm 51.89 \\
F2 & 2557 & \pm 223.9 & 2206 & \pm 172.2 \\
F3 & 3365 & \pm 212.7 & 3826 & \pm 316.6 \\
F3 & 4482 & \pm 161.8 & 3812 & \pm 485.3 \\
\hline
\end{tabular}


Tabla 3

Promedios y desviaciones estándar (DE) para FO, F1, F2, F3 y F4, para la vocal/i/ en hombres y mujeres de Santiago de Chile

\begin{tabular}{ccccc}
\hline & & & & \\
\hline Característica acústica & & Fomenino & & Masculino \\
\cline { 2 - 5 } de la voz & Promedio & $D E$ & Promedio & $D E$ \\
\hline F0 & 220 & \pm 21.82 & 153 & \pm 42.70 \\
F1 & 321 & \pm 52.35 & 308 & \pm 45.85 \\
F2 & 2684 & $\pm 224,2$ & 2360 & \pm 161.0 \\
F3 & 3568 & \pm 447.1 & 3125 & \pm 374.1 \\
F3 & 4413 & \pm 471.3 & 3921 & \pm 191.4 \\
\hline
\end{tabular}

Tabla 4

Promedios y desviaciones estándar (DE) para FO, F1, F2, F3 y F4, para la vocal /o/ en hombres y mujeres de Santiago de Chile

\begin{tabular}{|c|c|c|c|c|}
\hline \multicolumn{5}{|c|}{ Vocal /o/ } \\
\hline \multirow{2}{*}{$\begin{array}{c}\text { Característica acústica } \\
\text { de la voz }\end{array}$} & \multicolumn{2}{|c|}{ Femenino } & \multicolumn{2}{|c|}{ Masculino } \\
\hline & Promedio & $D E$ & Promedio & $D E$ \\
\hline FO & 216 & \pm 13.30 & 142 & \pm 34.89 \\
\hline F1 & 565 & \pm 376.8 & 497 & \pm 74.2 \\
\hline F2 & 1608 & \pm 829.7 & 1255 & \pm 688 \\
\hline F3 & 3331 & \pm 385.7 & 2834 & \pm 402.7 \\
\hline F3 & 4360 & \pm 109.2 & 3852 & \pm 448.7 \\
\hline
\end{tabular}

Tabla 5

Promedios y desviaciones estándar (DE) para FO, F1, F2, F3 y F4, para la vocal /u/ en hombres y mujeres de Santiago de Chile

\begin{tabular}{|c|c|c|c|c|}
\hline \multicolumn{5}{|c|}{ Vocal /u/ } \\
\hline \multirow{2}{*}{$\begin{array}{c}\text { Característica acústica } \\
\text { de la voz }\end{array}$} & \multicolumn{2}{|c|}{ Femenino } & \multicolumn{2}{|c|}{ Masculino } \\
\hline & Promedio & $D E$ & Promedio & $D E$ \\
\hline FO & 217 & \pm 25.70 & 137 & \pm 28.52 \\
\hline F1 & 420 & \pm 69.40 & 392 & \pm 76.8 \\
\hline F2 & 1540 & \pm 864.4 & 1341 & \pm 792 \\
\hline F3 & 3242 & \pm 387.3 & 2933 & \pm 460.2 \\
\hline F3 & 4081 & \pm 590.0 & 3934 & \pm 578.6 \\
\hline
\end{tabular}

Las diferencias porcentuales obtenidas al comparar los resultados de los universitarios chilenos con los obtenidos por Aronson et al. (2000) en Argentina con sujetos del Río de la Plata, tanto en mujeres como en hombres, se presentan en las Tablas 6 a 15 .

Tabla 6

Promedios de FO, F1, F2, F3 y F4, para la vocal /a/ en mujeres de Río de la Plata (2000) y Santiago (2012)

\begin{tabular}{cccc}
\hline & Vocal /a/ mujeres & \\
\hline Características acústicas de la voz & Santiago, Chile & Río del Plata, Argentina & Diferencia \% \\
\hline F0 & 214 & 205 & 4,4 \\
F1 & 697 & 330 & 110,9 \\
F2 & 1596 & 1553 & 2,8 \\
F3 & 2993 & 2890 & 3,6 \\
F4 & 4301 & 3930 & 9,4 \\
\hline
\end{tabular}


Tabla 7

Promedios de FO, F1, F2, F3 y F4, para la vocal /e/ en mujeres de Río de la Plata (2000) y Santiago (2012)

\begin{tabular}{cccc}
\hline & Vocal /e/ mujeres & \\
\hline Características acústicas de la voz & Santiago, Chile & Río del Plata, Argentina & Diferencia \% \\
\hline F0 & 216 & 205 & 5,4 \\
F1 & 486 & 330 & 47,1 \\
F2 & 2557 & 2500 & 2,3 \\
F3 & 3365 & 3130 & 7,5 \\
F4 & 4482 & 4150 & 8,0 \\
\hline
\end{tabular}

Tabla 8

Promedios de FO, F1, F2, F3 y F4, para la vocal /i/ en mujeres de Río de la Plata (2000) y Santiago (2012)

\begin{tabular}{cccc}
\hline & Vocal /i/ mujeres & & \\
\hline Características acústicas de la voz & Santiago, Chile & Río del Plata, Argentina & Diferencia \% \\
\hline F0 & 220 & 207 & 6,3 \\
F1 & 362 & 330 & 9,8 \\
F2 & 3030 & 2765 & 9,6 \\
F3 & 3568 & 3740 & $-4,6$ \\
F4 & 4413 & 4366 & 1,1 \\
\hline
\end{tabular}

Tabla 9

Promedios de FO, F1, F2, F3 y F4, para la vocal /o/ en mujeres de Río de la Plata (2000) y Santiago (2012)

\begin{tabular}{cccc}
\hline \multicolumn{4}{c}{ Vocal /o/ mujeres } \\
\hline Características acústicas de la voz & Santiago, Chile & Río del Plata, Argentina & Diferencia \% \\
\hline F0 & 216 & 204 & 5,7 \\
F1 & 565 & 546 & 3,5 \\
F2 & 1608 & 934 & 72,2 \\
F3 & 3331 & 2966 & 12,3 \\
F4 & 4360 & 3854 & 13,1 \\
\hline
\end{tabular}

Tabla 10

Promedios de FO, F1, F2, F3 y F4, para la vocal /u/ en mujeres de Río de la Plata (2000) y Santiago (2012)

\begin{tabular}{cccc}
\hline & Vocal /u/ mujeres & \\
\hline Características acústicas de la voz & Santiago, Chile & Río del Plata, Argentina & Diferencia \% \\
\hline F0 & 217 & 204 & 6,4 \\
F1 & 420 & 382 & 9,9 \\
F2 & 1540 & 740 & 108,1 \\
F3 & 3242 & 2760 & 17,5 \\
F4 & 4081 & 3380 & 20,7 \\
\hline
\end{tabular}

Tabla 11

Promedios de FO, F1, F2, F3 y F4, para la vocal /a/ en hombres de Río de la Plata (2000) y Santiago (2012)

\begin{tabular}{cccc}
\hline & Vocal /a/ hombres & & \\
\hline Características acústicas de la voz & Santiago, Chile & Río del Plata, Argentina & Diferencia \% \\
\hline F0 & 140 & 127 & 10,2 \\
F1 & 812 & 830 & $-2,2$ \\
F2 & 1332 & 1350 & $-1,3$ \\
F3 & 2774 & 2450 & 13,2 \\
F4 & 3986 & 3665 & 8,8 \\
\hline
\end{tabular}


Tabla 12

Promedios de FO, F1, F2, F3 y F4, para la vocal /e/ en hombres de Río de la Plata (2000) y Santiago (2012)

\begin{tabular}{cccc}
\hline & Vocal /e/ hombres & & \\
\hline Características acústicas de la voz & Santiago, Chile & Río del Plata, Argentina & Diferencia \% \\
\hline F0 & 164 & 125 & 31,3 \\
F1 & 474 & 430 & 10,2 \\
F2 & 2206 & 2120 & 4,1 \\
F3 & 2926 & 2628 & 11,3 \\
F4 & 3812 & 3610 & 5,6 \\
\hline
\end{tabular}

Tabla 13

Promedios de FO, F1, F2, F3 y F4, para la vocal /i/ en hombres de Río de la Plata (2000) y Santiago (2012)

\begin{tabular}{cccc}
\hline \multicolumn{4}{c}{ Vocal /i/ hombres } \\
\hline Características acústicas de la voz & Santiago, Chile & Río del Plata, Argentina & Diferencia \% \\
\hline F0 & 153 & 130 & 17,8 \\
F1 & 308 & 290 & 6,1 \\
F2 & 2360 & 2295 & 2,8 \\
F3 & 3125 & 2915 & 7,2 \\
F4 & 3921 & 3645 & 7,6 \\
\hline
\end{tabular}

Tabla 14

Promedios de FO, F1, F2, F3 y F4, para la vocal /o/ en hombres de Río de la Plata (2000) y Santiago (2012)

\begin{tabular}{cccc}
\hline & Vocal /o/ hombres & \\
\hline Características acústicas de la voz & Santiago, Chile & Río del Plata, Argentina & Diferencia \% \\
\hline F0 & 142 & 124 & 14,2 \\
F1 & 497 & 510 & $-2,6$ \\
F2 & 1255 & 860 & 46,0 \\
F3 & 2834 & 2480 & 14,3 \\
F4 & 3852 & 3485 & 10,5 \\
\hline
\end{tabular}

Tabla 15

Promedios de FO, F1, F2, F3 y F4, para la vocal /U/ en hombres de Río de la Plata (2000) y Santiago (2012)

\begin{tabular}{cccc}
\hline & Vocal /u/ hombres & & \\
\hline Características acústicas de la voz & Santiago, Chile & Río del Plata, Argentina & Diferencia \% \\
\hline F0 & 137 & 124 & 10,5 \\
F1 & 392 & 335 & 16,9 \\
F2 & 1341 & 720 & 86,3 \\
F3 & 2933 & 2380 & 23,2 \\
F4 & 3934 & 3355 & $-12,6$ \\
\hline
\end{tabular}

Por último, la comparación de los resultados obtenidos en el presente estudio con los expuestos por León (1998) en Concepción se presenta en las

Tablas 16, 17 y 18 . Al respecto, es importante señalar que el trabajo de León se refiere solo a la dispersión de las vocales anteriores y no considera la variable género. Por ello, para efectuar la comparación fue necesario utilizar valores promedios de F1 y F2 de las vocales /a/, /e/, /i/ y neutralizar la variable género, promediando los valores de hombres y mujeres.

Tabla 16

Promedios de F1 y F2 de vocal /a/ para Santiago de Chile y Concepción

\begin{tabular}{cccc}
\hline \multicolumn{5}{c}{ Vocal /a/ Santiago-Concepción } \\
\hline Formantes & Santiago, Chile & Concepción, Chile & Diferencia \% \\
\hline F1 & 755 & 730 & 3,4 \\
F2 & 1465 & 1400 & 4,6 \\
\hline
\end{tabular}


Tabla 17

Promedios de F1 y F2 de vocal /e/ para Santiago de Chile y Concepción

\begin{tabular}{cccc}
\hline \multicolumn{4}{c}{ Vocal /e/ Santiago-Concepción } \\
\hline Formantes & Santiago, Chile & Concepción, Chile & Diferencia \% \\
\hline F1 & 480 & 360 & 33,3 \\
F2 & 2382 & 2400 & $-0,8$ \\
\hline
\end{tabular}

Tabla 18

Promedios de F1 y F2 de vocal/i/ para Santiago de Chile y Concepción

\begin{tabular}{cccc}
\hline \multicolumn{5}{c}{ Vocal /i/ Santiago-Concepción } \\
\hline Formantes & Santiago, Chile & Concepción, Chile & Diferencia \% \\
\hline F1 & 315 & 280 & 12,5 \\
F2 & 2522 & 2800 & $-9,9$ \\
\hline
\end{tabular}

\section{Discusión y conclusiones}

La comparación entre las características de las vocales entre hombres y mujeres residentes en Santiago evidenció que las mujeres presentaron una mayor frecuencia que los hombres en F0, F1, F2, F3 y F4, excepto en el primer formante (F1) de la vocal /a/ donde la frecuencia era menor.

Al analizar el promedio de F0 de las cinco vocales en mujeres, se obtiene un promedio mayor en la vocal /i/, con $220 \mathrm{~Hz}$ y un promedio menor en las vocales /e/ y /o/ con $216 \mathrm{~Hz}$. En hombres, en cambio, se observa un mayor promedio en la vocal /e/ con $164 \mathrm{~Hz}$ y también un menor promedio en la vocal /u/ con $137 \mathrm{~Hz}$. Todos los promedios obtenidos para F0, F1, F2, F3, y F4 presentan una mayor frecuencia en mujeres, excepto en F1 de la vocal /a/ donde se aprecia un promedio levemente superior en hombres, ya que se obtuvo $812 \mathrm{~Hz}$ en el sexo masculino y $697 \mathrm{~Hz}$ en el sexo femenino. Este fenómeno se explicaría por una mayor apertura bucal.

Por otra parte, hombres y mujeres coinciden al presentar una mayor frecuencia en la vocal /a/ y una menor frecuencia en la vocal /i/. También se observa una coincidencia entre hombres y mujeres en F3, que resultó ser el formante con menor frecuencia para ambos sexos.

Al comparar los resultados con el estudio del español rioplatense, se evidenció que tanto los hombres como las mujeres de Santiago presentan una FO mayor que la de los hombres y mujeres de Río de la Plata. Las mayores diferencias en mujeres se observan en F1 de /a/ y /e/ y en el F2 de /o/ y /u/, mientras que en hombres las diferencias más importantes se encontraron en F2 de /o/ y /u/.

Al centrar la comparación en las mujeres, se advirtió que en la vocal /a/, se observaron resultados muy similares entre las hablantes de Santiago y las de Río de la Plata, con diferencias que no superan 10\%. Sin embargo, para la misma vocal en F1 se observa una diferencia porcentual que alcanza un $110,9 \%$, siendo superior F1 para las mujeres de Santiago. El primer formante (F1), se relaciona con la apertura de la cavidad bucal, por lo que esta diferencia podría deberse a que las mujeres de Santiago realizan una mayor apertura bucal al producir este fonema.

En la vocal /e/, también se observaron resultados similares entre los valores de Santiago y Río de la Plata, de hecho para F0, F2, F3 y F4 las diferencias no 
superan el $8 \%$. Sin embargo, para la misma vocal, en el $F 1$, se observa una diferencia porcentual que alcanza un $47,1 \%$. En esta vocal, se observa un fenómeno similar que el detectado en la vocal /a/, según el cual nuevamente podría atribuirse una mayor apertura bucal de las mujeres de Santiago.

En la vocal /i/, se advierten escasas diferencias porcentuales entre las características acústicas de Santiago y de Río de la Plata. De hecho, se observan diferencias que no superan el $10 \%$, de lo que se deduce que las mujeres de la ciudad de Santiago y las rioplatenses producirían dicho fonema de similar forma.

En la vocal /o/, por su parte, se aprecia una importante diferencia en F2, la cual alcanza un $72,2 \%$, siendo mayor el valor del formante en las mujeres de Santiago. F2 se vincula con la posición del cuerpo de la lengua, así las mujeres residentes en Santiago anteriorizarían más la lengua en la producción de este fonema respecto de las mujeres de Río de la Plata. Por otra parte, protruirían o redondearían menos los labios que las mujeres rioplatenses, por lo tanto estas últimas alargarían más su tracto vocal al producir dicho fonema. En la vocal /o/ también se observaron diferencias de un $13,2 \%$ y un $12,3 \%$ en F4 y F3, respectivamente. Debido a que F3 se relaciona con la posición de la punta de la lengua y el velo del paladar, se puede deducir que las mujeres de Santiago lo producirían con la punta de la lengua más anteriorizada y baja en relación con las rioplatenses, entonces, emitirían este fonema con el velo del paladar más descendido que las mujeres de Río de la Plata.

Por último, en la vocal /u/, las mayores diferencias las presenta F2 con un $108,1 \%$ y lo siguen
F4 y $\mathrm{F} 3$, con un $20,7 \%$ y un $17,5 \%$ respectivamente. De hecho, se observó el mismo fenómeno que en la vocal /o/, con proporciones similares. Los resultados obtenidos en $\mathrm{F} 2$ podrían atribuirse a que las mujeres residentes en Santiago, anteriorizarían más la lengua en la producción del fonema /u/, en relación con las mujeres de Río de la Plata, esto es, protruirían o redondearían menos los labios al producir la vocal /u/ que las mujeres del estudio argentino, por lo tanto estas últimas alargarían más su tracto vocal al producir dicho fonema. Mientras que los resultados para F3, se relacionarían con la posición de la punta de la lengua y el velo del paladar. Al respecto, se puede deducir que las mujeres residentes en la ciudad de Santiago producirían este fonema con la punta de la lengua más anteriorizada y baja en relación con las rioplatenses, emitiendo este fonema con el velo del paladar más descendido que las mujeres de Río de la Plata.

Adicionalmente, se pudo observar que F0, siempre, en todas las vocales, alcanzó un valor mayor en las mujeres residentes en la ciudad de Santiago, por lo tanto las mujeres de esta ciudad tendrían un tono habitual mayor que las mujeres de Río de la Plata.

En cuanto a la comparación de la producción de vocales entre los hombres de Santiago y los de Río de la Plata se pudo observar que en la vocal /a/ existían varias diferencias importantes, siendo estos últimos quienes presentan los valores más altos. La mayor diferencia porcentual es la de F3, que alcanzó un 13,2\%. Debido a que F3, tiene relación con la posición de la punta de la lengua y el velo del paladar, se puede deducir que los hombres residentes en la ciudad de Santiago, producirían este 
fonema con la punta de la lengua más anteriorizada y baja en relación con los rioplatenses, así como también con el velo del paladar más descendido. La segunda diferencia más importante se observó en F0, con un $10,2 \%$, lo que se relaciona con el número de ciclos de apertura y cierre glótico en un segundo y que, a su vez, determina el tono habitual promedio de los sujetos, el cual en este estudio resulta ser más agudo en los sujetos que viven en Santiago que el de aquellos que residen en Río de la Plata.

En esta vocal /a/, se destacan las siguientes diferencias: F0 con un $31,3 \%$, F3 con un $11,3 \%$ y F1 con un $10,2 \%$, siendo siempre los valores mayores los obtenidos por los sujetos chilenos de la ciudad de Santiago. De lo anterior, se puede deducir que los hombres de Santiago tendrían una mayor FO, es decir, un tono habitual más agudo que el de sus pares de Río de la Plata.

Al producir la vocal /e/, también presentan un F3 mayor debido a que emitirían este fonema con la punta de la lengua más posteriorizada y elevada en relación con los rioplatenses, esto es, los hombres residentes en Santiago producirían /e/ con el velo del paladar más descendido respecto de los hombres de Río de la Plata, Argentina.

La tercera diferencia importante está en F1, que es un 10,2\% mayor en los varones de Santiago. De ello se deduce que los hombres santiaguinos producirían el fonema /e/ con la cavidad bucal más abierta que los rioplatenses.

Por otra parte, al comparar los resultados de la vocal /i/, se observa solo una diferencia en F0 que alcanza a un $17,8 \%$, con valores mayores en los sujetos de Santiago. De este hallazgo, se desprende que los hombres de Santiago emitirían el fonema más agudo que sus congéneres de Río de la Plata.

En la vocal /o/, se aprecian diferencias porcentuales importantes en todas las características comparadas, excepto en F1, siendo siempre mayores los valores para los sujetos residentes en Santiago. La mayor diferencia (46\%) se observa en F2, seguido de F3 con un $14,3 \%$, F1 con un $14,2 \%$ y F4 con un $10,5 \%$. La diferencia en F2 se puede explicar, probablemente, porque los hombres de Santiago anteriorizarían más la lengua en la producción de este fonema, así como también protruirían o redondearían menos los labios que los hombres rioplatenses, por lo tanto estos últimos alargarían más su tracto vocal al producir dicho fonema. La diferencia en F3 puede explicarse probablemente porque los varones de Santiago producirían /o/ con la punta de la lengua más anteriorizada y baja, y también con el velo del paladar más descendido. Por último, se encontraron diferencias de un $14,2 \%$ en F0, lo que permite señalar que los sujetos de Santiago producen este fonema con un tono habitual más agudo que el de los rioplatenses.

Finalmente, al comparar las características obtenidas en la vocal /u/, se aprecian diferencias importantes en todas las características estudiadas. La mayor discrepancia en la vocal / $\mathrm{u} /$ se advierte en F2 (86,3\%), lo que implicaría que los hombres residentes en Santiago, anteriorizarían más la lengua en la producción de este fonema y que también protruirían o redondearían menos los labios que los hombres de Río de la Plata. Por consiguiente, estos últimos alargarían más su tracto vocal al producir dicho fonema. Además, en la emisión del fonema /u/ se observaron diferencias de un $23,2 \%$ en F3. Debido a que F3 se relaciona con la posición de la punta de 
la lengua y el velo del paladar, se puede deducir que los hombres de Santiago producirían este fonema con la punta de la lengua más anteriorizada y baja en relación con los rioplatenses, y también con el velo del paladar más descendido. Así, los hombres santiaguinos realizan una mayor apertura bucal al producir este fonema respecto de sus pares rioplatenses.

También se pudo observar que F0, en todas las vocales, alcanzó un valor mayor en los hombres residentes en Santiago, por lo tanto este grupo tendría un tono habitual mayor que los de Río de la Plata, es decir, tendrían la voz más aguda.

La comparación entre las vocales emitidas por los hablantes de Santiago y los de Concepción en Chile evidenció que en la vocal /a/ prácticamente no se observaron diferencias en F1 y F2, por ende los sujetos de ambos estudios tenderían a producir el fonema con un similar grado de apertura bucal y el cuerpo de su lengua adoptaría una posición parecida

En el caso de la vocal /e/, el valor promedio de F1 del estudio realizado en Concepción fue de $360 \mathrm{~Hz}$, en tanto que el promedio de F1 del efectuado en Santiago fue de $480 \mathrm{~Hz}$. Se observa así un 33\% de diferencia entre ambos promedios. El que el promedio de F1 sea superior en los hablantes de
Santiago indicaría una mayor apertura bucal en ellos. En cuanto a la diferencia entre los promedios en F2, esta fue de $-0.8 \%$, por lo tanto los sujetos de ambos estudios tenderían a producir este fonema con el cuerpo de la lengua en una posición similar y con un redondeamiento labial muy parecido .

En relación con la vocal /i/, en F1 no se observó una diferencia importante entre ambos estudios, ya que esta ascendería solo a un $12,5 \%$ con mayores valores para los hablantes santiaguinos. Esta discrepancia podría indicar una mayor apertura bucal de los sujetos del estudio realizado en Santiago. En F2 la diferencia es de un $-9,9 \%$ y podría implicar una mayor anteriorización del cuerpo de la lengua y una menor protrusión labial en los sujetos de Concepción

En general, se puede concluir entonces que no se advierten grandes diferencias entre las emisiones vocálicas de los fonemas /a/, /e/ /i/ entre hablantes residentes en Santiago y en Concepción, en Chile.

Evidentemente, las diferentes metodología dificultan la comparación entre los estudios, y la generalización de los resultados, sin embargo, una descripción de la producción de las vocales aisladas como las que aquí se presenta puede aportar al conocimiento de las características espectrográficas de ellas en nuestro país. 


\section{Referencias}

Aronson, L., Rufiner, H. L., Furmanski, H. y Estienne P. (2000). Características acústicas de las vocales del español rioplatense. Revista Fonoaudiológica, 46(2), 12-20.

Brain, A., Cepeda, G. y Barrientos, A. (1992a). Análisis sonográfico de /e/ en sílaba trabada (estrato alto de Valdivia, Chile). Estudios Filológicos, 27, 43-58.

Brain, A., Cepeda, G. y Barrientos, A. (1992 b). Análisis espectrográfico de la /a/ en el español de Valdivia, Chile. Revista de Filología, 13, 37-59.

Cepeda, G., Barrientos, A., Brain, A. y Miranda, J. C. (1991). La alofonía de /e/ en el estrato alto de Valdivia: análisis sonográfico en sílaba abierta. Estudios Filológicos, 26, 8398.

León, H. (1998). Determinación del campo de dispersión auditiva de las vocales de la serie anterior del español de Chile. Revista de Lingüística Teórica y Aplicada, 36, 87-96.

Soto Barba, J. (2007). Variación del F1 y del F2 en las vocales del español urbano y rural de la provincia de Ñuble. Revista de Lingüística teórica y Aplicada, 45(2), 143165.

Urrutia, H. (1976). Análisis fónico del español en el sur de Chile: los segmentos vocálicos átonos y tónicos (provincia de Valdivia). Estudios Filológicos, 8, 246-302. 